\title{
Is Early-onset Inflammatory Bowel Disease a Primary Immune Deficiency?
}

\author{
Rachna Shanbhag ${ }^{1}$, Sagar Bhattad ${ }^{2}$
}

\begin{abstract}
Pediatric-onset IBDs (PIBDs) are a group of genetically heterogeneous diseases with variable severity. Depending on the age of onset, inflammatory bowel disease (IBD) can be classified as pediatric-onset ( $<17$ years), early-onset ( $<10$ years), very early-onset ( $<6$ years), infant/toddler-onset ( $0-2$ years), and neonatal-onset IBD ( $<28$ days). Due to the advancement of molecular science and sequencing technologies, several monogenic defects have been identified in very early-onset IBD (VEO-IBD) over the last few decades. Inflammatory bowel disease manifesting at a very young age is more likely to be a monogenic defect. Pediatric-onset IBDs have been found to be associated with as many as 60 monogenic defects with most presenting below 6 years of age and some presenting before 1 year of age. The current article discusses early-onset IBD in detail with new concepts and a clinical and laboratory approach to these patients has been provided.

Keywords: Inflammatory bowel disease, Primary immune deficiency disease, Very early-onset IBD.

Pediatric Infectious Disease (2021): 10.5005/jp-journals-10081-1310
\end{abstract}

\section{INTRODUCTION}

The term inflammatory bowel disease (IBD) refers to chronic inflammation of the gut and encompasses a diverse group of disorders. Adult-onset IBD broadly includes two types - ulcerative colitis (UC) and Crohn's disease (CD). However, pediatric-onset IBD (PIBD) has distinct differences compared to adult-onset IBD.

\section{Pediatric-onset Inflammatory Bowel Disease}

Pediatric-onset IBDs (PIBD) are a group of genetically heterogeneous diseases with variable severity. Depending on the age of onset, IBD can be classified as pediatric-onset ( $<17$ years), early-onset ( $<10$ years), very early-onset ( $<6$ years), infant/toddler-onset $(0-2$ years), and neonatal-onset IBD ( $<28$ days). ${ }^{1}$ The incidence of PIBD in Asia and the Middle East varies from 0.5 to $11.4 / 100,000$ person. This is much lower than figures in Northern/Western Europe $(0.5-23 / 100,000)$ and North America (1.1-15.2/100,000). ${ }^{2}$

Inflammatory bowel disease presents in childhood in $25 \%$ of cases of which $1 \%$ present as early as in the neonatal or infantile period. According to a French study, very early-onset IBD (VEO-IBD) represents $3 \%$ of the total number of PIBDs. ${ }^{3}$

\section{Very Early-onset Inflammatory Bowel Disease}

Young children presenting with IBD-like features ( $<6$ years of age) are categorized as very early-onset inflammatory bowel disease (VEO-IBD). The incidence of VEO-IBD has been reported to be 4.37 per $100,000 .{ }^{4}$ Due to the advancement of molecular science and sequencing technologies, several monogenic defects have been identified in VEO-IBD over the last few decades. Many newer genetic defects are being added to this list (Table 1).

Compared to adults and PIBD, children with VEO-IBD have a more severe disease at presentation and are usually refractory to conventional immunosuppression. These children often present within the first few weeks to months of life with:
1,2Division of Pediatric Immunology and Rheumatology, Department of Pediatrics, Aster CMI Hospital, Bengaluru, Karnataka, India

Corresponding Author: Sagar Bhattad, Division of Pediatric Immunology and Rheumatology, Department of Pediatrics, Aster CMI Hospital, Bengaluru, Karnataka, India, Phone: +91 9779433934, e-mail: drsagarbhattad@gmail.com

How to cite this article: Shanbhag R, Bhattad S. Is Early-onset Inflammatory Bowel Disease a Primary Immune Deficiency? Pediatr Inf Dis 2021;3(3):125-127.

Source of support: Nil

Conflict of interest: None

- Bloody diarrhea.

- Mucoid stools.

- Weight loss.

- Abdominal pain.

- Perianal disease-fistulas, fissures, and abscess.

The earlier the age at onset of IBD, more stronger is the possibility of a monogenic cause. It is essential to perform genetic testing in VEO-IBD as a subset of these patients may be cured with hematopoietic stem cell transplantation (HSCT).

\section{Genetics of VEO-IBD}

Genetic predisposition plays an important role in the development of IBD in young children. ${ }^{5}$ Adult-onset IBD is polygenic and

Table 1: Discovery of monogenic defects in VEO-IBD

\begin{tabular}{ll}
\hline Genetic defect & Year of discovery \\
\hline IPEX & 1982 \\
IL-10 deficiency & 1993 \\
IL-10R deficiency & 2010 \\
RIPK-1 & 2018 \\
\hline
\end{tabular}

IL, interleukin; IPEX, X-linked immune dysregulation, polyendocrinopathy and enteropathy; RIPK-1, receptor-interacting serine/threonine-protein kinase 1

(0) The Author(s). 2021 Open Access This article is distributed under the terms of the Creative Commons Attribution 4.0 International License (https:// creativecommons.org/licenses/by-nc/4.0/), which permits unrestricted use, distribution, and non-commercial reproduction in any medium, provided you give appropriate credit to the original author(s) and the source, provide a link to the Creative Commons license, and indicate if changes were made. The Creative Commons Public Domain Dedication waiver (http://creativecommons.org/publicdomain/zero/1.0/) applies to the data made available in this article, unless otherwise stated. 
multifactorial in origin and these diseases are caused by an interplay of genetic susceptibility, environmental factors, and intestinal microbiota. On the other hand, IBD manifesting at a very young age, is more likely to be a monogenic defect. Pediatric-onset IBDs have been found to be associated with as many as 60 monogenic defects with most presenting below 6 years of age and some presenting before 1 year of age. ${ }^{3}$ Genes associated with VEO-IBD have been tabulated in Table 2.

\section{Pathogenesis of VeO-IBD}

The human gut is constantly exposed to antigens from the environment and the diet, and it harbors various commensal bacteria. IL-10, an anti-inflammatory cytokine, plays an important role in maintaining gut immune homeostasis. $T$ regulatory cells (Tregs) are a major source of IL-10 and play a crucial role in preventing unwarranted gut inflammation (Fig. 1). Hence, defects in Tregs and IL-10 pathway present with autoimmune enteropathy, causing VEO-IBD. ${ }^{6,7}$ While gut inflammation is the predominant manifestation of some PIDs (e.g., IL-10 deficiency), IBD-like disease can be an important associated manifestation in other PIDs [e.g., chronic granulomatous disease (CGD)].

\section{Evaluation of a Child with VEO-IBD}

The aim of evaluating children with VEO-IBD is to identify those patients who will benefit from non-conventional therapies and to identify those at risk of non-Gl complications. Evaluation includes a detailed history, including family history, physical examination,

Table 2: Monogenic causes of VEO-IBD

\begin{tabular}{ll}
\hline Genetic defects & Disease \\
\hline IL10 and IL1ORA, IL10RB & IL-10 signaling defect causing \\
FOXP3 & VEO-IBD \\
IL2RA & IPEX \\
ADAM17 & IPEX-like \\
IKBKG (encoding NEMO) & ADAM17 deficiency \\
& X-linked ectodermal immunode- \\
CYBB, CYBA, NCF1, NCF2, NCF4 & ficiency \\
G6PC3 & CGD \\
ITGB2 & Congenital neutropenia \\
SLC37A4 & LAD type I \\
$X I A P$ & Glycogen storage disease type Ib \\
CTLA4, LRBA & XLP type II \\
ICOS & Polyautoimmunity and com- \\
BTK & bined immune deficiency \\
CD40, CD4OL & CVID \\
ZAP70, IL2RG, ADA, RAG 1/2 & Agammaglobulinemia \\
WASP & Hyper lgM syndrome \\
STAT3 & SCID \\
MHCII & Wiskott-Aldrich syndrome \\
TTC7A & Hyper lgE syndrome (autosomal \\
& dominant) \\
\hline
\end{tabular}

IL, interleukin; IPEX, X-linked immune dysregulation, polyendocrinopathy, and enteropathy; CGD, chronic granulomatous disease; LAD, leukocyte adhesion defect; XLP, X-linked lymphoproliferative syndrome; CVID, common variable immunodeficiency; SCID, severe combined immunodeficiency endoscopic and histopathology evaluation, and genetic testing. A detailed history must include the age of onset, history of severe infections, skin involvement, and associated autoimmunity to arrive at a differential diagnosis. Family history contributes to the diagnosis if there is a history of IBD in family members and can also suggest autosomal recessive (AR), autosomal dominant (AD), or X-linked pattern (Table 3).

In the next section, we shall discuss a few cases and highlight how can one evaluate a child presenting with VEO-IBD.

\section{Case 1}

A 2-year-old boy born to a third-degree consanguineously married couple, presented with repeated episodes of bloody diarrhea from 6 months of age. He would strain while passing stools and had tenesmus. At 1 year of age, he developed a perianal abscess which was drained and treated with antibiotics. There was no history of pneumonia, recurrent ear, or skin infections. He had significant failure to thrive. He was evaluated by the pediatric gastroenterologist and upper and lower GI endoscopies were performed.

Upper GI endoscopy: Normal.

Lower GI endoscopy: Multiple ulcers were noted in the colonic mucosa.

Colonic biopsy: Cryptitis and crypt abscess suggestive of IBD.

\section{On Further Evaluation}

- Completeblood counts (CBC) did notshow anythrombocytopenia (Wiskott-Aldrich syndrome was unlikely).

- HIV card test: negative.

- Serum immunoglobulin was elevated.

- Nitroblue tetrazolium test (NBT) and dihydrorhodamine dye (DHR) tests: normal (CGD was excluded).

He was diagnosed to have IBD by the gastroenterologist but the early age of onset (6 months) warranted further evaluation. Genetic analysis (whole-exome sequencing) was performed by next-generation sequencing and a pathogenic mutation in the IL-10

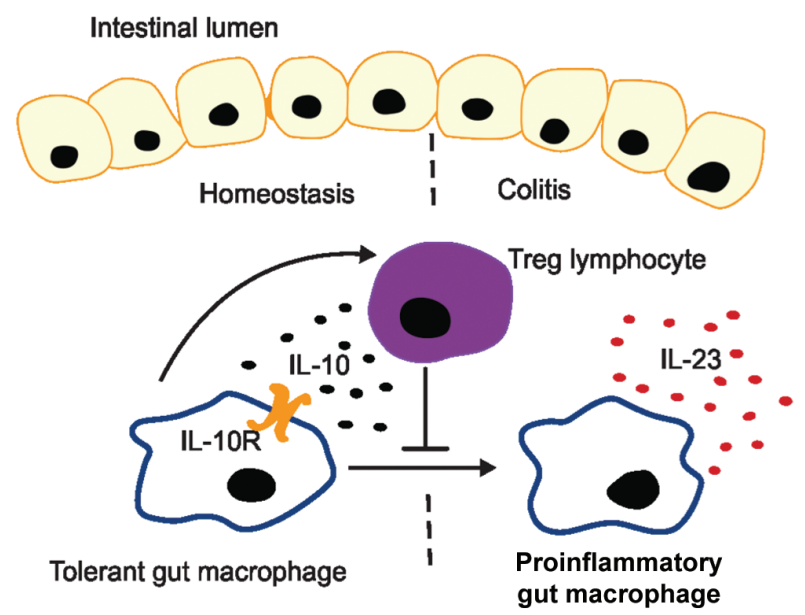

Fig. 1: Role of Tregs and IL-10 in the pathogenesis of VEO-IBD. [Macrophages play an important role in gut inflammation. Their proinflammatory potential is kept under check by the release of antiinflammatory cytokine (IL-10) by the Tregs. Failure of macrophages to respond to IL-10 gives rise to proinflammatory signals resulting in the development of colitis] 
Table 3: Evaluation in a child with VEO-IBD

\begin{tabular}{|c|c|c|}
\hline Presentation & Defect & Testing \\
\hline Neonatal onset, perianal disease-fistulas, diarrhea & IL-10, IL-10R deficiency & Genetic test* \\
\hline Male child, diarrhea, panenteric/perianal disease & $\mathrm{XLP}$ & Genetic testing for XIAP mutation \\
\hline $\begin{array}{l}\text { Male child, intractable diarrhea, eczematous/psoriasiform } \\
\text { dermatitis, polyendocrinopathy }\end{array}$ & IPEX & Genetic test for FOXP3 mutation \\
\hline $\begin{array}{l}\text { Chronic diarrhea, organomegaly, lymphadenopathy, autoim- } \\
\text { munity }\end{array}$ & IPEX-like (LRBA deficiency) & Genetic test \\
\hline $\begin{array}{l}\text { Severe infections, neutrophilic leukocytosis, thrombocytosis, } \\
\text { hypergammaglobulinemia, bloody diarrhea }\end{array}$ & CGD & NBT/DHR, genetic test \\
\hline $\begin{array}{l}\text { Male child, eczema, recurrent infections, thrombocytopenia } \\
\text { with small platelets, bloody diarrhea }\end{array}$ & WAS & Genetic test \\
\hline $\begin{array}{l}\text { Recurrent infections/non-healing ulcers, chronic diarrhea, } \\
\text { persistent neutrophilic leukocytosis }\end{array}$ & LAD & $\begin{array}{l}\text { CD18/CD11 expression flow cytometry, } \\
\text { genetic test }\end{array}$ \\
\hline
\end{tabular}

${ }^{*}$ Genetic test, exome sequencing study; IPEX, X-linked immune dysregulation, polyendocrinopathy and enteropathy; CGD, chronic granulomatous disease; WAS, Wiskott-Aldrich syndrome; LAD, leukocyte adhesion defect; NBT, Nitroblue tetrazolium test; DHR, Dihydrorhodamine dye, XLP, X-linked lymphoproliferative disease

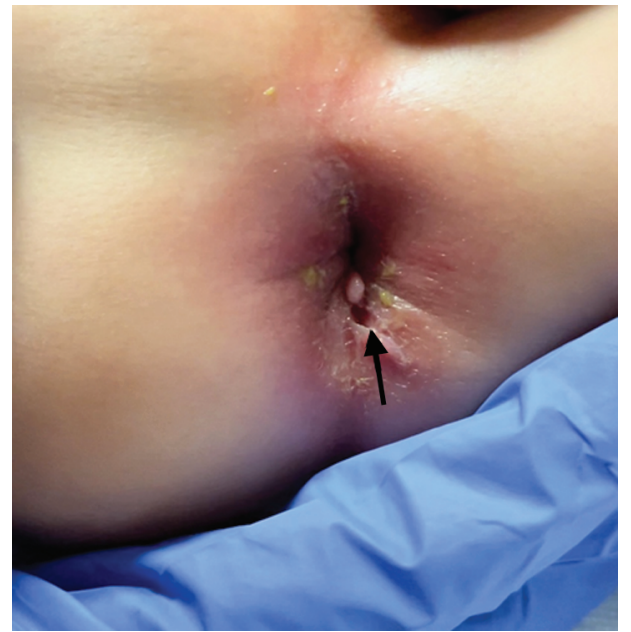

Fig. 2: Perianal fistula-manifestation of VEO-IBD in a 9-month-old boy

receptor gene was identified and the child underwent a successful bone marrow transplant.

\section{Diagnosis: VEO-IBD Due to IL-10R DEFECT}

\section{Case 2}

A 9-month-old boy presented with a history of repeated episodes of mucus-containing stools (10-12 episodes/day) since the age of 7 months. He would cry incessantly while passing stools. He was failing to thrive, though he had had no serious infections or hospitalizations. On examination, he was found to have perianal fistula and anal tag (Fig. 2).

\section{On Evaluation}

Complete blood counts: Hb-11 g/dL, TC-24,000/mm ${ }^{3}, \mathrm{~N}-60 \%, \mathrm{~L}-32 \%$, PC- $1,023,000 / \mathrm{mm}^{3}$.

Serum immunoglobulins: hypergammaglobulinemia.

Nitroblue tetrazolium test/DHR: abnormal, suggestive of impaired oxidative burst.
Diagnosis: VEO-IBD in a child with chronic granulomatous disease.

\section{Take Home Message}

- The onset of IBD below the age of 6 is called VEO-IBD and warrants detailed investigation.

- Very early-onset IBD is uncommon and accounts for $3 \%$ of PIBD.

- Very early-onset IBD is monogenic while adult-onset IBD is often polygenic.

- Very early-onset IBD must be investigated in detail including immunological and genetic evaluation.

- Hematopoietic stem cell transplantation is curable in certain forms of VEO-IBD, e.g., IL-10 and IL-10R defects.

\section{References}

1. Stein RE, Baldassano RN. Inflammatory bowel disease. In: St Geme K, Blum S, Tusker W, et al. Nelson textbook of pediatrics. 21st ed., vol. 1, Philadelphia: Elsevier; 2020. p. 1986.

2. Sýkora J, Pomahačová R, Kreslová $M$, et al. Current global trends in the incidence of pediatric-onset inflammatory bowel disease. World J Gastroenterol 2018;24(25):2741. DOI: 10.3748/wjg.v24.i25.2741.

3. Shim JO. Recent advance in very early onset inflammatory bowel disease. Pediatr Gastroenterol Hepatol Nutr 2019;22(1):41-49. DOI: 10.5223/pghn.2019.22.1.41.

4. Benchimol El, Fortinsky KJ, Gozdyra P, et al. Epidemiology of pediatric inflammatory bowel disease: a systematic review of international trends. Inflammat Bowel Dis 2011;17(1):423-439. DOI: 10.1002/ ibd.21349.

5. Doecke JD, Simms LA, Zhao ZZ, et al. Genetic susceptibility in IBD: overlap between ulcerative colitis and Crohn's disease. Inflammat Bowel Dis 2013;19(2):240-245. DOI: 10.1097/MIB.0b013e3182810041.

6. Paul G, Khare V, Gasche C. Inflamed gut mucosa: downstream of interleukin-10. Eur J Clin Investigat 2012;42(1):95-109. DOI: 10.1111/j.1365-2362.2011.02552.x.

7. Eastaff-Leung N, Mabarrack N, Barbour A, et al. Foxp3+ regulatory T cells, Th17 effector cells, and cytokine environment in inflammatory bowel disease. J Clin Immunol 2010;30(1):80-89. DOI: 10.1007/s10875009-9345-1. 